\title{
Pacific
}

Journal of

Mathematics

\section{ON THE ANALYTIC REFLECTION OF A MINIMAL SURFACE}

\section{JaIgyoung ChOe}

Volume 157 No. 1 


\title{
ON THE ANALYTIC REFLECTION OF A MINIMAL SURFACE
}

\author{
JAIGYOUNG CHOE
}

\begin{abstract}
For a long time it has been known that in a Euclidean space one can reflect a minimal surface across a part of its boundary if the boundary contains a line segment, or if the minimal surface meets a plane orthogonally along the boundary. The proof of this fact makes use of $\mathbf{H}$. A. Schwarz's reflection principle for harmonic functions.

In this paper we show that a minimal surface, as a conformal and harmonic map from a Riemann surface into $R^{3}$, can also be reflected analytically if it meets a plane at a constant angle.
\end{abstract}

THEOREM 1. Let $\Sigma \subset \mathbf{R}^{3}$ be a minimal surface with nonempty boundary $\partial \Sigma$ and let $\Pi$ be a plane. Suppose that $L \subset \Sigma \cap \Pi$ is a $C^{1}$ curve, $\Sigma$ is $C^{1}$ along $L$, and at all points of $L$ the tangent plane to $\Sigma$ makes a fixed angle $0<\theta<90^{\circ}$ with $\Pi$. Then $\Sigma$ can be analytically extended across $L$ to a minimal surface $\bar{\Sigma}$ satisfying the following properties:

(i) $\bar{\Sigma}=\Sigma \cup \Sigma^{*}$, where $\Sigma^{*}$ is the set of all images $p^{*}$ of $p \in \Sigma$ under an analytic reflection map * .

(ii) $p$ and $p^{*}$ are separated by $\Pi$ in such a way that

$$
\operatorname{dist}(p, \Pi)=\operatorname{dist}\left(p^{*}, \Pi\right) .
$$

(iii) The Gauss map $g: \bar{\Sigma} \rightarrow \mathbf{C}$ satisfies

$$
\overline{g(p)} \cdot g\left(p^{*}\right)=\left(\tan \frac{\theta}{2}\right)^{-2} \text {. }
$$

(iv) $p^{*} \in \Sigma^{*}$ is a branch point (geometric) if and only if $p \in \Sigma$ is.

(v) The map * is a single-valued immersion if $\Sigma$ is simply connected and $L$ is connected, or $\Sigma$ is doubly connected and $L$ is closed.

(vi) If * is single-valued, then $\Sigma^{*}$ has finite total curvature if and only if $\Sigma$ does.

(vii) If $\partial \Sigma=L$, then $\bar{\Sigma}$ is complete.

Proof. Let $x, y, z$ be coordinates of $\mathbf{R}^{3}$ such that $\Pi=\{(x, y, z)$ : $z=0\}$. Since $x, y, z$ are harmonic functions on the minimal surface $\Sigma$, one can find conjugate harmonic (possibly multiple-valued) 
functions $\bar{x}, \bar{y}, \bar{z}$ to $x, y, z$ respectively on $\Sigma$. Then

$$
u=x+i \bar{x}, \quad v=y+i \bar{y}, \quad w=z+i \bar{z}
$$

are holomorphic (possibly multiple-valued) functions on $\Sigma$, and

$$
d u=d x+i d \bar{x}, \quad d v=d y+i d \bar{y}, \quad d w=d z+i d \bar{z}
$$

are holomorphic 1-forms on $\Sigma$. Introduce $z, \bar{z}$ as conformal parameters on $\Sigma$. Then $\Sigma$ can be recaptured by setting

$$
x=\operatorname{Re} \int^{w} d u, \quad y=\operatorname{Re} \int^{w} d v, \quad z=\operatorname{Re} \int^{w} d w .
$$

From the conjugacy of $\bar{x}, \bar{y}, \bar{z}$ to $x, y, z$, it follows that

$$
d u^{2}+d v^{2}+d w^{2}=0 \text {. }
$$

Define a holomorphic differential $\omega$ and a meromorphic function $g$ on $\Sigma$ by

$$
\omega=d u-i d v, \quad g=\frac{d w}{d u-i d v} .
$$

Then we have

$$
\begin{aligned}
& x=\operatorname{Re} \int^{w} \frac{1}{2}\left(-g+\frac{1}{g}\right) d w, \\
& y=\operatorname{Re} \int^{w} \frac{i}{2}\left(g+\frac{1}{g}\right) d w, \\
& z=\operatorname{Re} \int^{w} d w .
\end{aligned}
$$

It is well known that $g$ is exactly the Gauss map of the surface $\Sigma$.

Put $-\Sigma=\{(x, y,-z):(x, y, z) \in \Sigma\}$ and define a Riemann surface $\widetilde{\Sigma}$ by $\widetilde{\Sigma}=\Sigma \cup(-\Sigma)$. For any $p=(x, y, z) \in \Sigma$, let $-p=(x, y,-z) \in-\Sigma$. Since $z=0$ on $\Sigma \cap(-\Sigma)$ ( $\supset L)$, we can extend the conformal parameters $z, \bar{z}$ over to $\widetilde{\Sigma}$ (across $L$ ) by the usual reflection with respect to $\Pi$, that is,

$$
z(-p)=-z(p) \text { and } \bar{z}(-p)=\bar{z}(p) \text { for any }-p \in-\Sigma \text {. }
$$

Hence we see that $d w$ is a well-defined holomorphic 1-form on the Riemann surface $\widetilde{\Sigma}$.

Now note that the constant angle hypothesis implies

$$
|g(p)|=\left(\tan \frac{\theta}{2}\right)^{-1} \text { for all } p \in L .
$$

In other words, $g$ maps $L$ into a circle in $\mathbf{C}$. Since $\Sigma$ is $C^{1}$ along $L$ and $L$ plays the same role in the Riemann surface $\widetilde{\Sigma}$ as a line does 
in $\mathbf{C}$, we can extend the Gauss map $g$ holomorphically over to $\widetilde{\Sigma}$ (across $L$ ) as follows. Define an extension of $g$, still called $g$, by

$$
g(-p)=\left(\tan ^{2} \frac{\theta}{2} \cdot \overline{g(p)}\right)^{-1}, \quad-p \in-\Sigma
$$

Clearly $g$ is holomorphic on $-\Sigma$ and continuous on $\widetilde{\Sigma}$. Let $h: \mathbf{C} \rightarrow \mathbf{C}$ be a linear transformation which maps the circle $|w|=\left(\tan \frac{\theta}{2}\right)^{-1}$ onto the imaginary axis of $\mathbf{C}$. Then the real part of $h \circ g$ is continuous on $\widetilde{\Sigma}$ and harmonic on $\Sigma$ and $-\Sigma$. Moreover we have

$$
\begin{aligned}
& \operatorname{Re}[h \circ g(-p)]=\operatorname{Re}[h \circ g(p)]=0 \text { for } p \in L, \\
& \operatorname{Re}[h \circ g(-p)]=-\operatorname{Re}[h \circ g(p)] \text { for }-p \in-\Sigma
\end{aligned}
$$

Hence by the reflection principle we conclude that $h \circ g$ is holomorphic on $\widetilde{\Sigma}$, and so is $g$.

Using this extended map $g$, the extended 1 -form $d w$, and the Weierstrass representation formula (1), we can obtain the extended minimal surface $\bar{\Sigma}$. Here, for any $p \in \Sigma, p^{*}$ is determined by integrating (1) over a contour on $\widetilde{\Sigma}$ from a fixed point to $-p$. In case $\Sigma$ is multiply connected it may happen that the reflection map $*$ maps $p \in \Sigma$ to infinitely many points $p^{*} \in \Sigma^{*}$. Also we should discuss the case where $g(p)=0$ or $\infty$. At such a point $p, w$ cannot be a parameter of $\Sigma$. However $d w$ and $\frac{1}{g} \pm g$ have a zero and a pole of the same order respectively at $-p$ as well as $p$. Consequently $d u$ and $d v$ are holomorphic at $-p$ and thus $\Sigma^{*}$ is well defined in a neighborhood of $p^{*}$. This proves conclusion (i).

Conclusion (ii) follows from the symmetry of $-\Sigma$ to $\Sigma$ and the formula for $z$ in (1).

(2) implies (iii).

Suppose $p$ is a regular point. If the tangent plane to $\Sigma$ at $p$ is parallel to $\Pi$, then $d w=0$ at $p$. For this reason, $w$ is not a good conformal parameter near the point $p$. However, for any conformal parametrization in a neighborhood of $p$, the metric of the corresponding immersion is, by [BC],

$$
d s^{2}=\frac{1}{2}\left(1+|g|^{2}\right)^{2}|\omega|^{2}=\frac{1}{2}\left(|g|+|g|^{-1}\right)^{2}|d w|^{2}
$$


Hence the ratio between the metrics at $p$ and $-p$ is given by

$$
\begin{aligned}
\frac{d s^{2}(-p)}{d s^{2}(p)} & =\frac{\frac{1}{2}\left(\tan ^{-2} \frac{\theta}{2} \cdot|g|^{-1}+\tan ^{2} \frac{\theta}{2} \cdot|g|\right)^{2}|d w|^{2}}{\frac{1}{2}\left(|g|+|g|^{-1}\right)^{2}|d w|^{2}} \\
& =\left(\frac{\tan ^{2} \frac{\theta}{2} \cdot|g|+\tan ^{-2} \frac{\theta}{2} \cdot|g|^{-1}}{|g|+|g|^{-1}}\right)^{2} .
\end{aligned}
$$

Note here that this ratio depends not on the parametrization of $\Sigma$ but on the geometry of $\Sigma$. Furthermore one can easily show that

$$
\begin{aligned}
0 & <\min \left(\tan ^{2} \frac{\theta}{2}, \tan ^{-2} \frac{\theta}{2}\right) \\
& \leq \frac{d s(-p)}{d s(p)} \leq \max \left(\tan ^{2} \frac{\theta}{2}, \tan ^{-2} \frac{\theta}{2}\right)<\infty .
\end{aligned}
$$

Therefore $\Sigma^{*}$ is also regular at $p^{*}$. Since $\Sigma=\left(\Sigma^{*}\right)^{*}$ and $p=\left(p^{*}\right)^{*}$, we can obtain the converse similarly.

For (v), we note that in either case every contour in $\widetilde{\Sigma}$ is nullhomotopic or homotopic to a contour in $\Sigma$ and that no forms in formula (1) have real periods on $\Sigma$. Hence $*$ is single-valued and so, by (3), an immersion.

To prove (vi), we use a formula for the Gauss curvature of $\Sigma$ [BC]:

$$
K=-\left[\frac{4\left|g^{\prime}\right|}{|f|\left(1+|g|^{2}\right)^{2}}\right]^{2}
$$

The curvature ratio between $p$ and $-p$ is given by

$$
\frac{K(-p)}{K(p)}=\frac{\left[\frac{4\left|g^{\prime}\right|}{\tan ^{6} \frac{\theta}{2} \cdot|g|^{3}\left(1+\tan ^{-4} \frac{\theta}{2} \cdot|g|^{-2}\right)^{2}}\right]^{2}}{\left[\frac{4\left|g^{\prime}\right|}{|g|^{-1}\left(1+|g|^{2}\right)^{2}}\right]^{2}}=\frac{\tan ^{4} \frac{\theta}{2} \cdot\left(1+|g|^{2}\right)^{4}}{\left(1+\tan ^{4} \frac{\theta}{2} \cdot|g|^{2}\right)^{4}} .
$$

Therefore

$$
\begin{aligned}
0 & <\min \left(\tan ^{12} \frac{\theta}{2}, \tan ^{-4} \frac{\theta}{2}\right) \\
& \leq \frac{K(-p)}{K(p)} \leq \max \left(\tan ^{12} \frac{\theta}{2}, \tan ^{-4} \frac{\theta}{2}\right)<\infty,
\end{aligned}
$$

and the conclusion follows.

Finally it is not difficult to see that (vii) can be derived from (3). Thus the proof of the theorem is now complete. 
COROLlary. Let $\Sigma$ be a complete minimal surface of finite total curvature in $\mathbf{R}^{3}$. If an end $E$ of $\Sigma$ meets a plane along $\partial E$ at a constant angle, then $\Sigma$ is the catenoid.

Proof. From Theorem 1 it follows that $\bar{E}=E \cup E^{*}$ is a complete minimal surface of finite total curvature with two ends. $\bar{E}$ must then be the catenoid [L]. Obviously, by the unique continuation property of a minimal surface, we have $\bar{E}=\Sigma$.

Let $\Sigma$ be a minimal surface in $\mathbf{R}^{3}$ with Gauss map $g$. For any real number $0<r<\infty$, let us denote by $\Sigma_{r}$ the minimal immersion of $\Sigma$ into $\mathbf{R}^{3}$ defined by the formula

$$
\begin{aligned}
& x=\operatorname{Re} \int^{w} \frac{1}{2}\left(-r g+\frac{1}{r g}\right) d w, \\
& y=\operatorname{Re} \int^{w} \frac{i}{2}\left(r g+\frac{1}{r g}\right) d w, \\
& z=\operatorname{Re} \int^{w} d w .
\end{aligned}
$$

Then we see that every minimal surface can be deformed into a 1parameter family of minimal surfaces and that this deformation preserves the $z$-coordinate and multiplies the Gauss map by $r$.

THEOREM 2. Assume $\Sigma \subset \mathbf{R}^{3}$ is a minimal surface with nonempty boundary $\partial \Sigma$ which makes a constant angle $\theta$ with a plane $\Pi$ along $\partial \Sigma \cap \Pi$.

(i) For any real number $0<r<\infty$, the minimal surface $\Sigma_{r}$ makes a constant angle $\phi=2 \tan ^{-1}\left(\frac{1}{r} \tan \frac{\theta}{2}\right)$ with $\Pi$ along $\partial \Sigma_{r} \cap \Pi$.

(ii) There exists a positive real number s such that the minimal surface $\Sigma_{s}$ meets $\Pi$ orthogonally along $\partial \Sigma_{s} \cap \Pi$, and the analytic extension $\bar{\Sigma}$ of $\Sigma$ is the same as $\left(\Sigma_{s} \cup\left(\Sigma_{s}\right)^{*}\right)_{1 / s}$, where $\left(\Sigma_{s}\right)^{*}$ is the usual reflection (mirror image) of $\Sigma_{s}$ with respect to $\Pi$.

Proof. (i) By hypothesis, $|g(p)|=\left(\tan \frac{\theta}{2}\right)^{-1}$ for all $p \in \partial \Sigma \cap \Pi$. Then

$$
|r g(p)|=r\left(\tan \frac{\theta}{2}\right)^{-1}=\left(\tan \frac{\phi}{2}\right)^{-1},
$$

where $\phi=2 \tan ^{-1}\left(\frac{1}{r} \tan \frac{\theta}{2}\right)$. Since the deformation of $\Sigma$ into $\Sigma_{r}$ 
preserves the $z$-coordinate and multiplies the Gauss map by $r, \Sigma_{r}$ meets $\Pi$ along $\partial \Sigma_{r} \cap \Pi$ at the constant angle $\phi$.

(ii) Let $s$ be the positive real number satisfying

$$
2 \tan ^{-1}\left(\frac{1}{s} \tan \frac{\theta}{2}\right)=90^{\circ} \text {. }
$$

Then $\Sigma_{s}$ meets $\Pi$ orthogonally. Clearly we have

$$
(\bar{\Sigma})_{S}=\overline{\left(\Sigma_{S}\right)} \text {. }
$$

Since $\overline{\left(\Sigma_{s}\right)}$ is the union of $\Sigma_{s}$ and its mirror image $\left(\Sigma_{S}\right)^{*}$ with respect to $\Pi$, we conclude that

$$
\bar{\Sigma}=\left((\bar{\Sigma})_{s}\right)_{1 / s}=\left(\overline{\left(\Sigma_{s}\right)}\right)_{1 / s}=\left(\Sigma_{s} \cup\left(\Sigma_{s}\right)^{*}\right)_{1 / s} .
$$

REMARKS. 1. A nice example of the analytic reflection can be seen in the catenoid. Let $\Pi_{1}, \Pi_{2}$, and $\Pi_{3}$ be the parallel planes with $\operatorname{dist}\left(\Pi_{1}, \Pi_{2}\right)=\operatorname{dist}\left(\Pi_{2}, \Pi_{3}\right)$. Let $\Sigma$ be the catenoid whose ends are parallel to the $\Pi_{i}$. Then $\Sigma$ intersects the $\Pi_{i}$ along circles at constant angles $\alpha_{i}$. Assume $\alpha_{2} \neq 90^{\circ}$ and define $D_{1}, D_{3}$ to be the two bounded components of $\Sigma \sim\left(\Pi_{1} \cup \Pi_{2} \cup \Pi_{3}\right)$. Then $D_{3}$ is the analytic reflection of $D_{1}$ with respect to $\Pi_{2}$ and $D_{1}$ is that of $D_{3}$. If we define $D_{+}, D_{-}$to be the components of $\Sigma \sim \Pi_{2}$, then $D_{+}=\left(D_{-}\right)^{*}$ and $D_{-}=\left(D_{+}\right)^{*}$.

2. Embeddedness of $\Sigma$ does not necessarily imply that of $\Sigma^{*}$.

3. If the tangent plane to $\Sigma$ at $p$ is parallel to $\Pi$, so is the tangent plane to $\Sigma^{*}$ at $p^{*}$. This is clear in view of Theorem 1(iii).

4. Given an angle $0<\theta<90^{\circ}$, two points $p_{1}, p_{2}$ on $\Pi$, and a curve $\Gamma \subset \mathbf{R}^{3}$ from $p_{1}$ to $p_{2}$, one can construct an area minimizing surface $\Sigma$ with the fixed boundary $\Gamma$ and a free boundary $L \subset \Pi$ along which $\Sigma$ meets $\Pi$ at the angle $\theta$ as follows. Let $\Gamma_{1}$ be the line segment on $\Pi$ from $p_{2}$ to $p_{1}$. We regard $\Gamma, \Gamma_{1}$ as 1-dimensional sets with orientation, i.e., 1-currents. Let $S$ be a surface with $\partial S=$ $\Gamma \cup \Gamma_{S}, \Gamma_{S} \subset \Pi$. Give $S$ and $\Gamma_{S}$ orientations, $S$ is then called a 2-current, in such a way that $\partial S=\Gamma-\Gamma_{S}$. As sets, $\Gamma_{1}$ and $\Gamma_{S}$ bound a planar domain $D \subset \Pi$ with $\partial D=\Gamma_{1} \cup \Gamma_{S}$. Giving suitable orientations to each component of $D$, we can make $D$ into a 2-current such that $\partial D=\Gamma_{1}+\Gamma_{S}$. Let us fix an orientation of the plane $\Pi$. Then $D$, as a set, is divided into two disjoint domains $D_{1}, D_{2}$ such that $D_{1}$ and $D_{2}$ with the orientation inherited from $\Pi$ can be thought of as 2-currents, and

$$
D=D_{1}-D_{2}
$$


Now we define $\widetilde{A}(S)$, the modified area of $S$, by

$$
\widetilde{A}(S)=\operatorname{Area}(S)+\cos \theta\left[\operatorname{Area}\left(D_{1}\right)-\operatorname{Area}\left(D_{2}\right)\right] .
$$

Let $\mathscr{F}$ be the family of all 2-currents $S$ such that $\partial S-\Gamma$ is a 1current on $\Pi$. Then it is not difficult to see that $-\infty<\inf \{\widetilde{A}(S): S \in$ $\mathscr{F}\}$ and therefore we can find a modified area minimizing current $\Sigma$. $\Sigma$, as a set, is a desired minimal surface, and by [T] it is Hölder continuously differentiable up to its free boundary. Thus we can analytically extend $\Sigma$ across its free boundary $\partial \Sigma \sim \Gamma$ to obtain the $\theta$-reflection $\Sigma^{*}$ of $\Sigma$ with respect to $\Pi$.

Open problems. 1. Is it possible to extend Theorem 1 to the case of a constant mean curvature surface in $\mathbf{R}^{3}$ or a minimal hypersurface in $\mathbf{R}^{n}$ ? It is well known that the answer is yes if a constant mean curvature surface (a minimal hypersurface respectively) meets a plane (a hyperplane respectively) orthogonally.

2. As a generalization of Corollary, is it true that if a complete constant mean curvature surface $\Sigma$ of finite topological type intersects a plane at a constant angle $\neq 90^{\circ}$, then $\Sigma$ is a Delaunay's surface?

3. Given a compact convex body $U$ in $\mathbf{R}^{3}$, one can construct a minimal disk $D$ in $U$ which makes a constant contact angle $\theta$ with the convex boundary $\partial U$ ? Grüter and Jost [GJ] solved the problem affirmatively when $\theta=90^{\circ}$.

4. Most complete minimal surfaces are known to have at least one plane of symmetry. However, some complete immersed minimal surfaces of genus zero constructed by $\mathrm{H}$. Karcher do not have a plane of symmetry. Nevertheless, given a complete minimal surface in $\mathbf{R}^{3}$, can one find a plane which intersects the minimal surface at a constant angle?

Acknowledgment. This paper was written while I was visiting Bonn University through SFB 256 in the Summer 1989. I wish to express my gratitude to M. Grüter, S. Hildebrandt, and H. Karcher for their hospitality.

\section{REFERENCES}

[BC] L. Barbosa and G. Colares, Minimal Surfaces in $\mathbf{R}^{3}$, Springer, Berlin, 1986.

[GJ] M. Grüter and J. Jost, On embedded minimal disks in convex bodies, Analyse non linéaire, 3 no. 5, (1986), 345-390. 
[L] H. B. Lawson, Jr., Lectures on Minimal Submanifolds, vol. 1, Publish or Perish, Berkeley, 1980.

[T] J. E. Taylor, Boundary regularity for solutions to various capillarity and free boundary problems, Comm. Partial Differential Equations, 2 no. 4, (1977), 323-357.

Received December 20, 1989.

PosTECH

P.O. Box 125

Pohang, South Korea 


\title{
PACIFIC JOURNAL OF MATHEMATICS
}

Founded by

\author{
E. F. Beckenbach (1906-1982) F. Wolf (1904-1989)
}

\section{EDITORS}

V. S. VARADARAJAN

(Managing Editor)

University of California

Los Angeles, CA 90024-1555

vsv@math.ucla.edu

Herbert Clemens

University of Utah

Salt Lake City, UT 84112

clemens@math.utah.edu

F. Michael Christ

University of California

Los Angeles, CA 90024-1555

christ@math.ucla.edu

THOMAS ENRIGHT

University of California, San Diego

La Jolla, CA 92093

tenright@ucsd.edu
Nicholas ERcolani

University of Arizona

Tucson, AZ 85721

ercolani@math.arizona.edu

R. FInN

Stanford University

Stanford, CA 94305

finn@gauss.stanford.edu

VAughan F. R. Jones

University of California

Berkeley, CA 94720

vfr@math.berkeley.edu

STEVEN KERCKHOFF

Stanford University

Stanford, CA 94305

spk@gauss.stanford.edu

\section{C. MOORE}

University of California

Berkeley, CA 94720

MARTIN SCHARLEMANN

University of California

Santa Barbara, CA 93106

mgscharl@henri.ucsb.edu

Harold Stark

University of California, San Diego La Jolla, CA 92093

\section{SUPPORTING INSTITUTIONS}

UNIVERSITY OF ARIZONA

UNIVERSITY OF BRITISH COLUMBIA

CALIFORNIA INSTITUTE OF TECHNOLOGY

UNIVERSITY OF CALIFORNIA

MONTANA STATE UNIVERSITY

UNIVERSITY OF NEVADA, RENO

NEW MEXICO STATE UNIVERSITY

OREGON STATE UNIVERSITY
UNIVERSITY OF OREGON

UNIVERSITY OF SOUTHERN CALIFORNIA

STANFORD UNIVERSITY

UNIVERSITY OF HAWAII

UNIVERSITY OF TOKYO

UNIVERSITY OF UTAH

WASHINGTON STATE UNIVERSITY

UNIVERSITY OF WASHINGTON 


\section{PACIFIC JOURNAL OF MATHEMATICS}

Volume $157 \quad$ No. $1 \quad$ January 1993

Permutation enumeration symmetric functions, and unimodality

FRANCESCO BRENTI

On the analytic reflection of a minimal surface

JAIGYOUNG CHOE

Contractive zero-divisors in Bergman spaces

Peter Larkin DUREn, DMitry Khavinson, Harold SEymour

SHAPIRO and CARL SUNDBERG

On the ideal structure of positive, eventually compact linear operators on

Banach lattices

RUEY-JEN JANG and HAROLD DEAN VICTORY, JR.

A note on the set of periods for Klein bottle maps

JAUME LLIBRE

Asymptotic expansion at a corner for the capillary problem: the singular case

\section{ERICH MIERSEMANN}

A state model for the multivariable Alexander polynomial

JUN MURAKAMI

Free Banach-Lie algebras, couniversal Banach-Lie groups, and more

Vladimir G. Pestov

Four manifold topology and groups of polynomial growth

RICHARD ANDREW STONG

A remark on Leray's inequality

AKIRA TAKESHITA

$A_{\infty}$ and the Green function

JANG-MEI GLORIA WU

Integral spinor norms in dyadic local fields. I 